\title{
A Multicast-Traffic-Oriented Energy-Saving Algorithm with a Hybrid Sleep Mode for EPONs
}

\author{
Lincong Zhang, ${ }^{1}$ Lei Guo, ${ }^{2}$ Yejun Liu, ${ }^{2}$ Kefeng Wei, ${ }^{2,3}$ and Weigang Hou ${ }^{2}$ \\ ${ }^{1}$ School of Information Science and Engineering, Shenyang Ligong University, Shenyang 110159, China \\ ${ }^{2}$ College of Information Science and Engineering, Northeastern University, Shenyang 110819, China \\ ${ }^{3}$ Shenkan Engineering and Technology Corporation, MCC, Shenyang 110016, China \\ Correspondence should be addressed to Lei Guo; haveball@gmail.com
}

Received 29 October 2015; Accepted 19 January 2016

Academic Editor: Iraj Sadegh Amiri

Copyright (C) 2016 Lincong Zhang et al. This is an open access article distributed under the Creative Commons Attribution License, which permits unrestricted use, distribution, and reproduction in any medium, provided the original work is properly cited.

\begin{abstract}
High energy consumption in Ethernet Passive Optical Networks (EPONs) has caused intense research on energy-saving methods in recent years. The most common method for EPON energy saving is to cause optical network units (ONUs) to sleep which are idle or low loaded. With the popularity of multimedia applications, the ONUs carry not only unicast traffic but also more and more multicast traffic. However, existing studies mainly focus on ONUs that support only unicast traffic. The many differences between multicast traffic and unicast traffic make it necessary to design novel energy-saving methods. This paper proposes a multicasttraffic-oriented energy-saving algorithm for EPONs, called the energy-saving algorithm for multicast traffic (ESMT). To save as much energy as possible, the proposed algorithm uses a hybrid sleep mode (composed of a deep sleep state and an independent sleep mode) in which not only can ONU enter a deep sleep state, but also the ONU transmitter and receiver can sleep independently. Simulation results show that the proposed algorithm, which is oriented to multicast traffic in EPONs, is more energy efficient than other algorithms.
\end{abstract}

\section{Introduction}

In recent decades, the rapid development of modern industries has intensified the greenhouse effect and has threatened the survival and development of humankind. Broadband access networks, one of the last-mile solutions, consume about $75 \%$ of all network energy [1]. Therefore, decreasing the energy consumption of broadband access networks is an effective way to green all networks. Furthermore, the Ethernet Passive Optical Network (EPON) has become one of the most feasible broadband access technologies, for its low energy consumption and wide deployment [2,3]. Consequently, energy saving is a topic of paramount importance in EPON contexts today.

The typical EPON is structured as a tree topology, where the optical line terminal (OLT) acts as the root node and optical network units (ONUs) act as the leaf nodes. The splitter bridges the OLT and the ONUs through bidirectional optical fibers. Upstream, ONUs contend for the same shared wavelength channel using time division. Downstream, the OLT can support three kinds of traffic: unicast, multicast, and broadcast. The tree topology enables the EPON to be perfectly suitable for multicast and broadcast traffic. With the popularity of multimedia applications, more and more multicast traffic and unicast traffic are sharing the same ONU. As shown in Figure 1, the OLT keeps separate buffers for multicast and unicast traffic. The same-colored ONUs belong to the same multicast group and receive the same multicast traffic. All ONUs in a multicast group must be active simultaneously to receive the traffic designated for the group. This is the major difference between an EPON oriented to unicast traffic and one oriented to multicast traffic.

Both unicast and multicast traffic are broadcast by the OLT to all ONUs. The traffic can only be received by the destination ONUs and is discarded by the other irrelevant ONUs. Studies show that more than $95 \%$ of the traffic is discarded, which means a great deal of energy is wasted on unnecessary processing by the irrelevant ONUs. Therefore, 


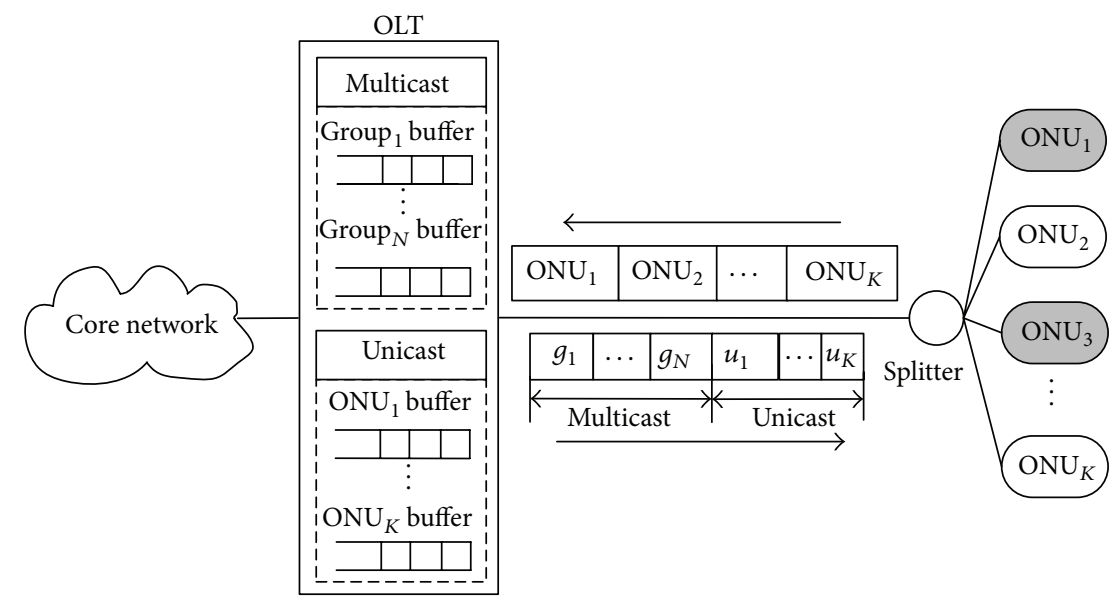

FIgURE 1: EPON multicast and unicast traffic setup.

the irrelevant ONUs stay in a low-power or sleep state to save energy. However, most literature focuses on energy saving oriented to unicast traffic [4-18]. In this work, we propose an energy-saving algorithm for multicast traffic (ESMT) to reduce the energy consumption of EPON ONUs oriented to multicast traffic, while maintaining the traffic delay at an acceptable level.

Our ESMT considers not only downstream traffic, but also upstream traffic. ONUs in the same group must receive the downstream multicast traffic at the same time; however, the upstream transmissions of these ONUs cannot proceed simultaneously. Therefore, the ONU hybrid sleep mode presented in our previous work [5] is a better way to solve the energy-saving problem in this situation. In other words, the ONU receivers in the same group can sleep simultaneously after the reception; meanwhile, the ONU transmitters can sleep independently, according to their own idle time. If there is no upstream or downstream traffic, the ONU goes into a deep sleep state. However, due to multicast characteristics, the energy saving of ONUs oriented to multicast traffic in hybrid sleep mode is much more complicated than in [5].

To the best of our knowledge, there is no prior study concentrating on energy saving that considers both upstream and downstream multicast traffic. More importantly, because it makes the ONU transmitter and receiver sleep separately, our ESMT algorithm is more energy efficient than other algorithms.

The remainder of this paper is organized as follows. Section 2 overviews related work. In Section 3, we formulate the problem of minimizing ONU energy consumption. Section 4 illustrates the proposed ESMT algorithm. In Section 5, we detail the simulation results. Finally, Section 6 concludes the paper.

\section{Related Work}

In existing work, ONU energy saving can be realized by making part of the ONU elements sleep by dynamic bandwidth allocation (DBA). Yin et al. [15] studied the stability of predictor-based dynamic bandwidth allocation over EPON; bandwidth utilization can be improved by adaptive control at the OLT. The ONU dozing mode, specified in the standard ITU-T G.984 for Gigabit PON (GPON), saves energy by turning off the ONU transmitter when there is no upstream data to transmit [16]. Zhang et al. [17] proposed a sleep control scheme composed of downstream traffic scheduling rules at the OLT and sleep control rules at ONUs, which reduces energy consumption and improves bandwidth utilization effectively. Dhaini et al. [8] proposed a green bandwidth allocation (GBA) framework to achieve the maximum possible energy saving. In GBA, hybrid cyclic/deep sleep is enabled. After sending the request message to and receiving a grant message from the OLT, each ONU remains idle for a period of time, during which the ONU can enter an OLT-based or ONU-based deep sleep mode. The doze mode happens after the ONU-based deep sleep mode in order to receive a grant message.

Dias and Wong $[10,11]$ demonstrated that energy saving can be achieved when the idle time of a vertical-cavity surface-emitting laser (VCSEL) ONU is more than the sleepto-active transition time. The VCSEL ONU can turn into sleep or doze modes during its idle time. In particular, the VCSEL ONU will enter sleep mode when its idle time is more than 2 ms or turn into doze mode when its idle time is between $330 \mathrm{~ns}$ and $2 \mathrm{~ms}$. Energy-saving methods in these mentioned works are based on a combination of existing sleep modes. However, these methods are not sufficiently flexible because the upstream and downstream transmissions do not finish at the same time. Thus, in [5], we presented the independent sleep mode for ONUs, in which the transmitter and receiver of the ONU can sleep independently according to upstream and downstream traffic, respectively. Hosseinabadi and Ansari [18] also considered independent sleep of the transmitter and the receiver by dividing nine states for ONU in different situations. Both upstream and downstream traffic are taken into consideration.

Furthermore, all the above works only present energysaving strategies for unicast traffic. Because of the differences between unicast and multicast traffic, the sleep processes of ONUs oriented to multicast traffic cannot be handled 
by the strategies in [4-18]. The authors in [19-21] mainly studied the bandwidth allocation problem of multicast traffic in TDM-PON (time division multiplexing-PON), WDMPON (wavelength division multiplexing-PON), and TDMWDM-PON, respectively. However, they did not consider the energy-saving issue. Currently, only a few previous energysaving methods are oriented to multicast traffic in EPONs. Valcarenghi et al. [22, 23] proposed an energy-efficient multicasting solution by setting the ONUs belonging to the groups which were not receiving data into sleep mode. The ONU sleep mode is combined with optimal multicast group scheduling to reduce the wake-up overhead and energy consumption. However, the author only considered downstream traffic. In fact, putting the ONUs into sleep mode should take into account both upstream and downstream traffic to ensure the Quality of Service (QoS). This is exactly the focus of this paper.

\section{System Model}

In this section, we define the system model in terms of the ONU sleep mode, the energy consumption model, and the problem formulation to minimize ONU energy consumption.

3.1. Sleep Mode. Downstream, ONUs belonging to the same multicast group can receive the multicast traffic simultaneously. However, the upstream channel is shared by all ONUs, in the way of time division multiple access (TDMA); that is, at any time, only the polled ONU can transmit its upstream traffic. Therefore, the other unpolled ONUs' transmitters are idle. In other words, if we utilize the traditional sleep mode, in which the ONUs do not sleep unless there is no upstream or downstream traffic, unnecessary energy will be wasted. Consequently, the sleep processes of ONUs oriented to multicast traffic become more complicated. However, more energy can be saved by using the hybrid sleep mode of transmitter and receiver, which was presented in our previous work [5]. The transmitter and receiver sleep independently according to their own traffic. As illustrated in Figure 2, $\mathrm{ONU}_{1}, \mathrm{ONU}_{2}$, and $\mathrm{ONU}_{3}$ belong to the same group and their receivers sleep simultaneously after time $t_{3}$. Upstream, we assume that the ONUs transmit their data by ascending order of their own index numbers. Therefore, $\mathrm{ONU}_{1}$ transmitter begins to sleep at time $t_{1}$. Similarly, $\mathrm{ONU}_{2}$ transmitter sleeps before time $t_{1}$ and after time $t_{2}$. $\mathrm{ONU}_{3}$ transmitter sleeps before time $t_{2}$ and after time $t_{4}$. As a result, $\mathrm{ONU}_{1}$ and $\mathrm{ONU}_{2}$ can enter deep sleep mode at time $t_{3}$, and $\mathrm{ONU}_{3}$ can enter deep sleep mode at time $t_{4}$. Since the hybrid sleep mode in [5] can save more energy than traditional sleep modes, this result is also appropriate for ONUs oriented to multicast traffic. In other words, we efficiently combine the characteristics of multicast traffic and hybrid sleep mode, intending to minimize the energy consumption of ONUs with both upstream and downstream traffic (including unicast and multicast traffic).

3.2. Energy Consumption Model. Since we utilize the hybrid sleep mode, we can divide the ONU into three parts: the transmitter, the receiver, and the common modules [24]. The common modules refer to the parts that always keep working, even if the ONU goes into deep sleep mode. Certainly, these parts also work when the transmitter sends upstream traffic or the receiver receives downstream traffic. The notations related to ONU energy consumption are shown in Notations.

The energy consumption of each ONU in Figure 2 is represented in Figure 3. Since Figure 2 only shows the sleep process in one cycle, we are not sure whether to wake up the ONU at the beginning of the next cycle. Hence, the overhead time $T_{\text {ov }}$ is drawn by a dotted line, and $P_{\text {wake-up }}$ is not marked in Figure 3. The ONU energy consumption can be composed of four parts, namely, energy consumption of the transmitter, energy consumption of the receiver, energy consumption of the common modules, and energy consumption produced by the ONU wake-up process.

We use $E^{i}$ to denote the energy consumption of $\mathrm{ONU}_{i}$. Therefore, $E^{i}$ can be formulated as follows:

$$
\begin{aligned}
E^{i} & =E_{\mathrm{tx}}^{i}+E_{\mathrm{rx}}^{i}+E_{\text {sleep }}^{i}+E_{\text {wake-up }}^{i}, \\
E_{\mathrm{tx}}^{i} & =t_{\mathrm{tx}}^{i} \cdot P_{\mathrm{tx}}, \\
E_{\mathrm{rx}}^{i} & =t_{\mathrm{rx}}^{i} \cdot P_{\mathrm{rx}}, \\
E_{\text {sleep }}^{i} & =t_{\mathrm{sim}}^{i} \cdot P_{\text {sleep }}, \\
E_{\text {wake-up }}^{i} & =n_{\mathrm{wake-up}}^{i} \cdot T_{\mathrm{ov}} \cdot P_{\text {wake-up }},
\end{aligned}
$$

where $E_{\mathrm{tx}}^{i}, E_{\mathrm{rx}}^{i}$, and $E_{\text {sleep }}^{i}$ denote the energy consumed by the transmitter and the receiver of $\mathrm{ONU}_{i}$ during their active time and the common modules of $\mathrm{ONU}_{i}$ during the running time, respectively. $E_{\text {wake-up }}^{i}$ denotes the energy consumption of $\mathrm{ONU}_{i}$ during its wake-up process from deep sleep mode. The $\mathrm{ONU}_{i}$ wake-up process consists of clock recovery and synchronization. $E_{\text {wake-up }}^{i}$ is large enough that it cannot be omitted. Hence, researchers are engaged in reducing the time and energy consumption of clock recovery and synchronization [3]. Frequent wake-ups significantly increase the ONU energy consumption. Hence, it is necessary to reduce the ONUs' wake-up times.

3.3. Problem Formulation. Based on the hybrid sleep mode, we attempt to minimize the total energy consumption of all ONUs. Therefore, the objective of this paper can be defined as the minimum energy consumption (MEC) problem. For simplicity in problem formulation, we introduce the following notations:

$N, J$ : the set of all ONUs and multicast groups, respectively.

$i$ : index number of ONUs, $1 \leq i \leq|N|$.

$j$ : index number of multicast groups, $1 \leq j \leq|J|$.

$h$ : index number of upstream transmission slots, $1 \leq$ $k \leq|N|$.

$k$ : index number of downstream transmission slots, $1 \leq k \leq|J|$. 


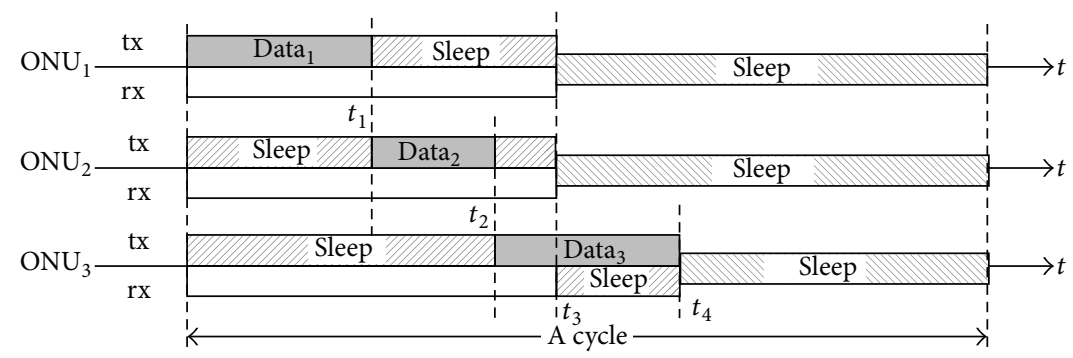

FIGURE 2: Sleep processes of ONUs with both upstream traffic and downstream multicast traffic.

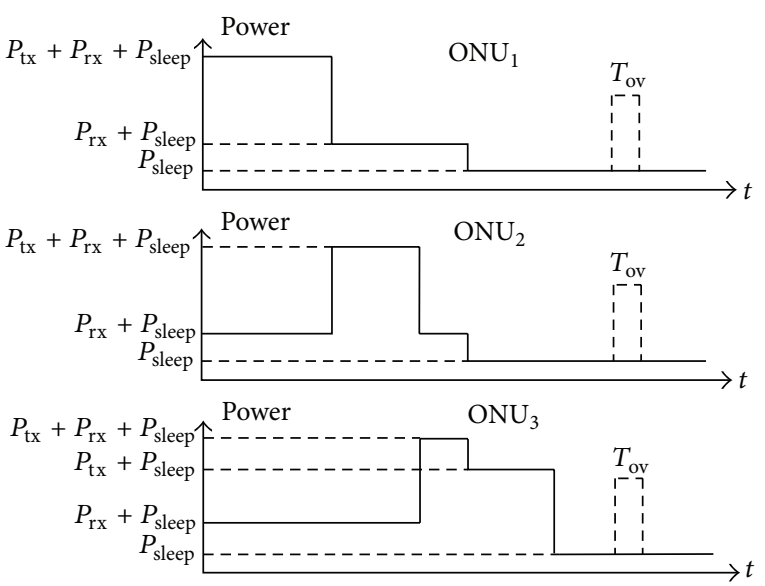

FIgURE 3: Power consumption of each ONU in Figure 2.

$W$ : matrix with $|N|$ rows and $|J|$ columns.

$\alpha_{i}^{k}$ : binary variable, taking 1 if the receiver of $\mathrm{ONU}_{i}$ is active in $k_{\mathrm{th}}$ downstream transmission slot and 0 otherwise.

$\beta_{i}^{h}$ : binary variable, taking 1 if the transmitter of $\mathrm{ONU}_{i}$ is active in $h_{\mathrm{th}}$ upstream transmission slot and 0 otherwise.

$\xi_{i}^{k}$ : binary variable, taking 1 if $\mathrm{ONU}_{i}$ needs to wake up at the beginning of $k_{\mathrm{th}}$ downstream transmission slot and 0 otherwise.

$\omega_{i, j}$ : binary variable, taking 1 if $\mathrm{ONU}_{i}$ is subscribed to multicast group $j$ and 0 otherwise.

We assume that there are $|N|$ upstream transmission slots. Each slot is assigned to an ONU, and the length of the slot is the transmission time of this ONU's upstream traffic. We also assume that there are $|J|$ downstream transmission slots, and each downstream transmission slot is assigned to a multicast group. In other words, $k_{\mathrm{th}}$ downstream transmission slot is assigned to multicast group $j$, where $k=j$; and the length of $k_{\mathrm{th}}$ downstream transmission slot is the transmission time of the downstream traffic of multicast group $j$. It is worth noting that unicast traffic can be seen as multicast traffic of a multicast group with only one member. Hence, the energy consumption of $\mathrm{ONU}_{i}$ in (1) can also be represented as follows:

$$
\begin{aligned}
E^{i} & =\sum_{k=0}^{|J|-1}\left(\alpha_{i}^{k} \cdot P_{\mathrm{rx}} \cdot t_{\mathrm{rx}}^{i k}+\beta_{i}^{h} \cdot P_{\mathrm{tx}} \cdot t_{\mathrm{tx}}^{i}+P_{\text {sleep }} \cdot t_{\text {sleep }}^{i}+\xi_{i}^{k}\right. \\
& \left.\cdot P_{\text {wake-up }} \cdot T_{\mathrm{ov}}\right),
\end{aligned}
$$

where $t_{\mathrm{rx}}^{i k}$ denotes the working period of $k_{\mathrm{th}}$ downstream transmission slot of the $\mathrm{ONU}_{i}$ receiver. As a result, the MEC problem can be formulated as follows:

$$
\begin{array}{ll}
\text { Minimize } & E^{i} \\
\text { Subject to } & \alpha_{i}^{k}=1, \quad \forall i: \omega_{i, j}=1, \quad \forall k \\
& \alpha_{i}^{k}-\alpha_{i}^{k-1} \leq \xi_{i}^{k}, \quad \forall i, \quad \forall k \\
& \sum_{h=0}^{|N|-1} \beta_{i}^{h}=1, \quad \forall i \\
& \sum_{i=1}^{|N|} \beta_{i}^{h}=1, \quad \forall h .
\end{array}
$$

The objective formulated in (7) is to minimize the energy consumption of $\mathrm{ONU}_{i}$. The constraint in (8) ensures that all ONU receivers belonging to the same multicast group must stay active during the transmission of multicast traffic for the multicast group. The constraint in (9) determines whether to wake up the ONU at the beginning of $k_{\mathrm{th}}$ downstream transmission slot. The constraint in (10) ensures that each ONU can stretch across one, and only one, upstream transmission slot. The constraint in (11) enables each upstream transmission slot to be assigned to only one ONU. To solve the MEC problem, we propose the ESMT algorithm described in the next section.

\section{Energy-Saving Algorithm for Multicast Traffic (ESMT)}

Due to the transmission characteristics of upstream and downstream traffic, this paper introduces the hybrid sleep mode-that is, the combination of independent sleep mode and deep sleep mode mentioned in Section 3.1-to solve the sleep problem of ONUs oriented to multicast traffic in 


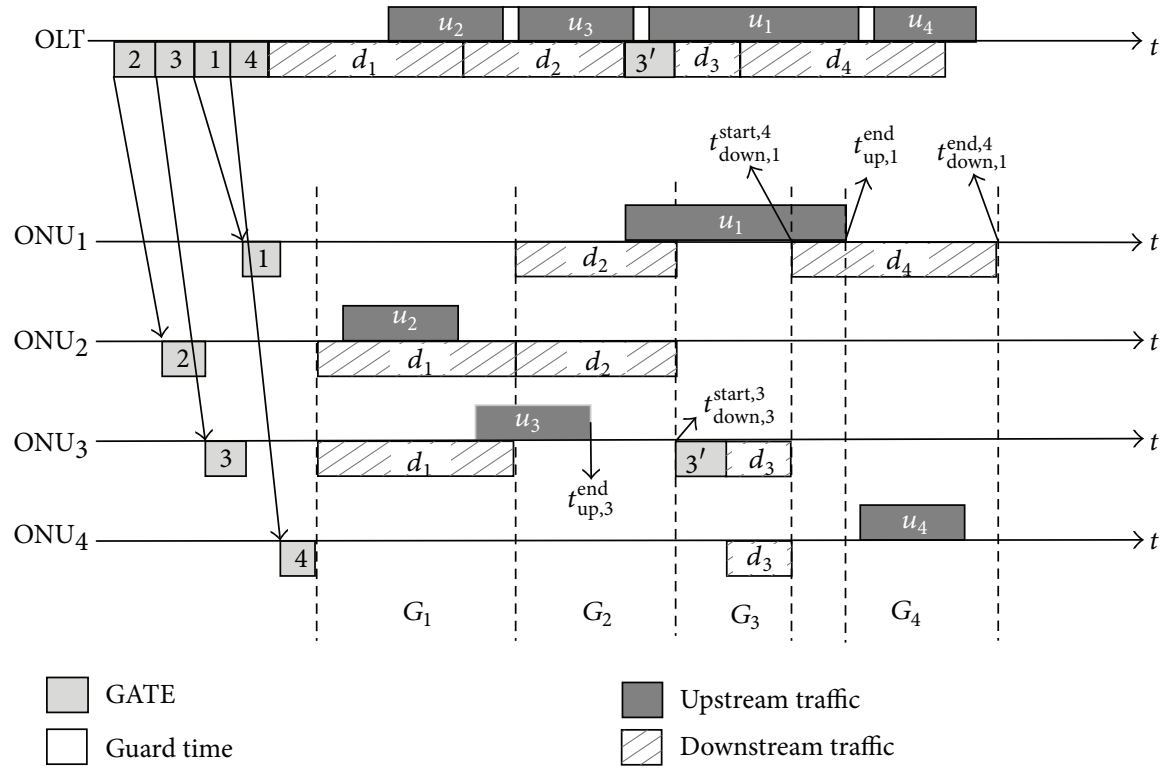

FIGURE 4: Sequence chart of EPON traffic scheduling.

EPONs. Based on the hybrid sleep mode, we propose the ESMT algorithm.

In this paper, $j_{\text {th }}$ multicast group is denoted by $G_{j}(n$, $\left.O_{j}[n]\right)$, where $n$ denotes the number of ONUs contained in $j_{\text {th }}$ multicast group and $O_{j}[n]$ is used to store the index numbers of the ONUs in $j_{\text {th }}$ multicast group. For simplicity, we use $G_{j}$ to represent $G_{j}\left(n, O_{j}[n]\right)$. Each multicast group stretches across only one slot, and the slot length depends on the traffic transmission time of the multicast group that stretches across this slot. Furthermore, each slot can only carry the downstream traffic of one multicast group. In addition, we consider downstream unicast traffic as a particular kind of multicast traffic where $n=1$.

Figure 4 shows the traffic scheduling in one cycle. The first multicast group contains two ONUs, that is, $\mathrm{ONU}_{2}$ and $\mathrm{ONU}_{3}$. Hence, we can obtain the first multicast group $G_{1}\left(2, O_{1}[2]\right)$, where $O_{1}[2]=\{2,3\}$. Similarly, we can obtain the second multicast group $G_{2}\left(2, O_{2}[2]\right)$, where $O_{2}[2]=$ $\{1,2\}$, the third multicast group $G_{3}\left(2, O_{3}[2]\right)$, where $O_{3}[2]=$ $\{3,4\}$, and the fourth multicast group $G_{4}\left(1, O_{4}[1]\right)$, where $O_{4}[1]=\{1\}$. We can see that the OLT first sends a GATE control message to each ONU, in which the upstream transmission slot, sleep start time, and wake-up time for each ONU are included. Therefore, before sending the GATE control message, the OLT should obtain the information about transmission slots for both upstream and downstream traffic. Especially downstream, a certain ONU may receive downstream traffic in successive slots, when sending a GATE control message makes the computation for the ONU's sleep and wake-up processes much more complicated.

To determine the sleep processes of the ONUs' transmitters, we sort all ONUs in a multicast group by ascending order of their own index numbers. Therefore, we can obtain $\{2,3\}$, $\{1,2\},\{3,4\}$, and $\{1\}$ from all the sequential multicast groups. Then, removing the nonoverlapping indices in sequence from the four number sets, we get the upstream transmission sort as $2 \rightarrow 3 \rightarrow 1 \rightarrow 4$.

Since the wake-up processes consume considerable energy that cannot be neglected, we must be aware of the wake-up times for each ONU. First, the start time and the end time of both upstream and downstream transmissions should be determined. Then, the ONUs' wake-up times can be counted. The entire process is described in the following steps.

Step 1. Compute the start time and the end time of the upstream transmission. Let $S_{m}$ denote the index number of $m_{\text {th }}$ ONU that is sending upstream traffic; then, we need to compute the start time $t_{\mathrm{up}, S_{m}}^{\text {start }}$ and the end time $t_{\mathrm{up}, S_{m}}^{\text {end }}$ for each $\mathrm{ONU}_{S_{m}}$ as $t_{\text {up }, S_{m+1}}^{\text {start }}=t_{\text {up }, S_{m}}^{\text {start }}+B_{S_{m}}^{\text {up }} / R+T_{\text {guard }}$ and $t_{\text {up }, S_{m}}^{\text {end }}=$ $t_{\text {up } S_{m}}^{\text {start }}+B_{S_{m}}^{\text {up }} / R$, where $B_{S_{m}}^{\text {up }}$ denotes the allocated bandwidth of $\mathrm{ONU}_{S_{m}}, T_{\text {guard }}$ is the guard time between two successive transmissions, and $R$ is the transmission rate. Thus, we can obtain the start time $t_{\mathrm{up}, i}^{\text {start }}$ and end time $t_{\mathrm{up}, i}^{\mathrm{end}}$ of the upstream transmission of $\mathrm{ONU}_{i}(1 \leq i \leq N)$. Based on these two time points, we can determine the sleep time of the $\mathrm{ONU}_{i}$ transmitter and compute the sleep start and end time of the $\mathrm{ONU}_{i}$ transmitter of [5].

Step 2. Compute the start time $t_{\text {down }}^{\text {start } k}$ and the end time $t_{\text {down }}^{\text {end } k}$ for each downstream transmission slot as $t_{\text {down }}^{\text {end, }}=t_{\text {down }}^{\text {start } k}+$ $L^{k} / 10^{9}$ and $t_{\text {down }}^{\text {start }, k+1}=t_{\text {down }}^{\text {end }, k}$, where $L^{k}$ denotes the traffic load of $k_{\text {th }}$ downstream transmission slot. If $\mathrm{ONU}_{i}$ belongs to $j_{\text {th }}$ $(j=k)$ multicast group, we use $t_{\mathrm{down}, i}^{\mathrm{star},}$ and $t_{\mathrm{down}, i}^{\mathrm{end},}$ to denote the start time and the end time of $\mathrm{ONU}_{i}$ in $k_{\text {th }}$ downstream transmission slot. Based on these two time points, we can determine the $\mathrm{ONU}_{i}$ receiver's sleep time and compute its sleep start and end time [5]. 


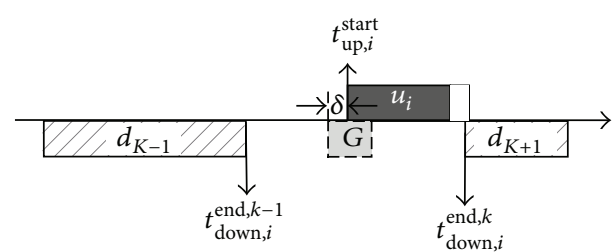

FIGURE 5: Start time of the $\mathrm{ONU}_{i}$ upstream transmission between two downstream transmissions.

Step 3. At the beginning of each polling cycle, the OLT will send a GATE control message to each $\mathrm{ONU}_{i}$, according to the order obtained above. Hence, the wake-up times of each $\mathrm{ONU}_{i}$ are at least equal to 1 . In addition to the wake-up process at the beginning of each polling cycle, whether $\mathrm{ONU}_{i}$ wakes up at the beginning of the other downstream transmission slots within the cycle is determined by the following situations.

(1) The end time of the $\mathrm{ONU}_{i}$ upstream transmission is during $k_{\text {th }}$ downstream transmission slot, and $\mathrm{ONU}_{i}$ is in a deep sleep state between $t_{\text {up }, i}^{\text {end }}$ and $t_{\text {down }, i}^{\text {start } k+1}$. Take $\mathrm{ONU}_{3}$ in Figure 4 as an example and let $\Delta_{\text {idle }}=t_{\text {down, } 3}^{\text {start, }}-t_{\mathrm{up}, 3}^{\text {end }}-T_{\mathrm{ov}}$. As the receiver has entered the sleep state before $t_{\text {up }, 3}^{\text {end }}$, only the transmitter and the common modules consume energy during $\Delta_{\text {idle }}$. Assuming that $\mathrm{ONU}_{3}$ goes into deep sleep state during $\Delta_{\text {idle }}$, its saved energy during $\Delta_{\text {idle }}$ can be computed as $\Delta_{\text {idle }} \cdot\left(P_{\mathrm{tx}}+P_{\text {sleep }}\right)-\Delta_{\text {idle }} \cdot P_{\text {sleep }}=\Delta_{\text {idle }} \cdot P_{\mathrm{tx}}$. Therefore, if and only if both $\Delta_{\text {idle }}>T_{\mathrm{ov}}$ and $\Delta_{\text {idle }} \cdot P_{\mathrm{tx}}>T_{\mathrm{ov}}$. $P_{\text {wake-up }}$ (the energy consumption constraint) are met, $\mathrm{ONU}_{3}$ can enter deep sleep state during $\Delta_{\text {idle }}$ and wake up before the transmission of downstream data $d_{3}$, and the wake-up times increase by 1 . Consequently, we need to insert a GATE control message into the downstream queue to enable $\mathrm{ONU}_{3}$ to receive it before $d_{3}$ and update the start time and end time of all the downstream transmissions after $t_{\text {down }, 3}^{\text {start, }}$.

(2) As is shown in Figure 5, the start time of $\mathrm{ONU}_{i}$ 's upstream transmission is between two downstream transmissions; let $\Delta_{\text {idle }}=t_{\mathrm{up}, i}^{\mathrm{start}}-\delta-T_{\mathrm{ov}}-t_{\mathrm{down}, i}^{\mathrm{end}, k-1}$, where $\delta$ is the time used to abstract the useful information from the GATE control message. As the transmitter has entered the sleep state between $t_{\mathrm{down}, i}^{\mathrm{end}, k-1}$ and $t_{\mathrm{up}, i}^{\mathrm{star}}$, only the receiver and the common modules consume energy during $\Delta_{\text {idle }}$. Assuming that $\mathrm{ONU}_{i}$ goes into deep sleep state during $\Delta_{\text {idle }}$, the saved energy of $\mathrm{ONU}_{i}$ during $\Delta_{\text {idle }}$ can be computed as $\Delta_{\text {idle }} \cdot\left(P_{\mathrm{rx}}+P_{\text {sleep }}\right)-$ $\Delta_{\text {idle }} \cdot P_{\text {sleep }}=\Delta_{\text {idle }} \cdot P_{\text {rx }}$. Therefore, if and only if both $\Delta_{\text {idle }}>$ $T_{\text {ov }}$ and $\Delta_{\text {idle }} \cdot P_{\text {rx }}>T_{\text {ov }} \cdot P_{\text {wake-up }}$ (i.e., the energy consumption constraint) are met, $\mathrm{ONU}_{i}$ can enter deep sleep state during $\Delta_{\text {idle }}$ and wake up before the upstream transmission, and the wake-up times increase by 1 . Consequently, we need to insert a GATE control message into the downstream queue to enable $\mathrm{ONU}_{i}$ to receive it before the upstream transmission, which leads to $t_{\text {down, } i}^{\text {end }, k}=t_{\text {down, }, i}^{\text {end } k}+t_{G}$, where $t_{G}$ denotes the transmission time of a GATE control message. Then, update the start and end time of all the downstream transmissions after $t_{\mathrm{down}, i}^{\text {end }}$.

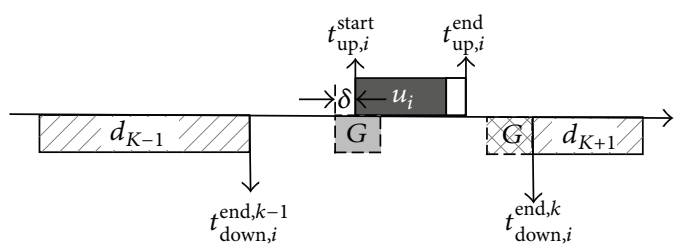

FIGURE 6: Both start time and end time of the $\mathrm{ONU}_{i}$ upstream transmission between two downstream transmissions.

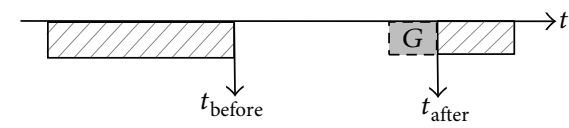

Figure 7: No upstream transmission between two downstream transmissions.

(3) As is shown in Figure 6, both the start time and end time of the $\mathrm{ONU}_{i}$ upstream transmission are between two downstream transmissions. We address the case before $t_{\mathrm{up}, i}^{\text {start }}$ following situation (2) and the case after $t_{\mathrm{up}, i}^{\text {end }}$ following situation (1). If both cases need to wake up $\mathrm{ONU}_{i}$, we insert a GATE control message for each case; the locations of the GATEs are shown in Figure 6. After inserting GATE control messages, we let $t_{\text {down }, i}^{\text {end } k}=t_{\text {down }, i}^{\text {end },}+2 t_{G}$ and update the start time and end time of all downstream transmissions after $t_{\text {down, }, \text { e }}^{\text {end }}$.

(4) As is shown in Figure 7, there is no upstream transmission between two downstream transmissions. We denote the end time of the first downstream transmission by $t_{\text {before }}$ and the start time of the second downstream transmission by $t_{\mathrm{after}}$ and let $\Delta_{\text {idle }}=t_{\mathrm{after}}-t_{\mathrm{before}}-T_{\mathrm{ov}}$. As the transmitter has entered the sleep state before $t_{\text {before}}$, only the receiver and the common modules consume energy during $\Delta_{\text {idle }}$. Assuming that $\mathrm{ONU}_{i}$ goes into deep sleep state during $\Delta_{\text {idle }}$, the saved energy of $\mathrm{ONU}_{i}$ during $\Delta_{\text {idle }}$ can be computed as $\Delta_{\text {idle }} \cdot\left(P_{\text {rx }}+P_{\text {sleep }}\right)-\Delta_{\text {idle }} \cdot P_{\text {sleep }}=\Delta_{\text {idle }} \cdot P_{\text {rx }}$. Therefore, if and only if both $\Delta_{\text {idle }}>T_{\text {ov }}$ and $\Delta_{\text {idle }} \cdot P_{\mathrm{rx}}>T_{\text {ov }} \cdot P_{\text {wake-up }}$ (i.e., the energy consumption constraint) are met, $\mathrm{ONU}_{i}$ can enter the deep sleep state during $\Delta_{\text {idle }}$ and wake up before $t_{\mathrm{after}}$, and the wake-up times increase by 1 . Consequently, we must insert a GATE control message into the downstream queue to enable $\mathrm{ONU}_{i}$ to receive it before $t_{\text {after }}$, which leads to $t_{\mathrm{after}}=t_{\mathrm{after}}+t_{\mathrm{G}}$. Then, update the start and end time of all downstream transmissions after $t_{\text {after }}$.

(5) As is shown in Figure 8, there is no downstream transmission between two upstream transmissions. We denote the end time of the first upstream transmission by $t_{\text {before }}$ and the start time of the second upstream transmission by $t_{\mathrm{after}}$ and let $\Delta_{\text {idle }}=t_{\text {after }}-\delta-T_{\mathrm{ov}}-t_{\text {before }}$. As the receiver has entered sleep state at $t_{\text {before}}$, only the transmitter and the common modules consume energy during $\Delta_{\text {idle }}$. Assuming that $\mathrm{ONU}_{i}$ goes into deep sleep state during $\Delta_{\text {idle }}$, the saved energy of $\mathrm{ONU}_{i}$ during $\Delta_{\text {idle }}$ can be computed as $\Delta_{\text {idle }} \cdot\left(P_{\text {tx }}+P_{\text {sleep }}\right)-$ $\Delta_{\text {idle }} \cdot P_{\text {sleep }}=\Delta_{\text {idle }} \cdot P_{\text {tx }}$. Therefore, if and only if $\Delta_{\text {idle }}>T_{\text {ov }}$ and $\Delta_{\text {idle }} \cdot P_{\mathrm{tx}}>T_{\mathrm{ov}} \cdot P_{\text {wake-up }}$ (i.e., the energy consumption constraint), $\mathrm{ONU}_{i}$ can enter deep sleep state during $\Delta_{\text {idle }}$ and wake up before $t_{\mathrm{after}}$, and the wake-up times increase by 1 . Consequently, we must insert a GATE control message into 


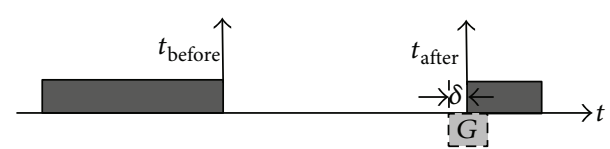

Figure 8: No downstream transmission between two upstream transmissions.

the downstream queue to enable $\mathrm{ONU}_{i}$ to receive it before $t_{\text {after }}$. Then, update the start and end time of all downstream transmissions after $t_{\text {after }}$.

In our proposed algorithm, the OLT takes charge of the upstream and downstream slot allocations and determines the wake-up times of each ONU. In addition to the downstream data, the OLT also sends GATE control messages to the ONUs, which contain the slot allocations and the information about sleep and wake-up. However, the ONU functions are much simpler than the OLT. The ONUs transmit upstream data and a REPORT control message with a bandwidth request to the OLT. In addition, the ONUs comply with the GATE control message to sleep or wake up.

\section{Simulation Results}

For simulation, we name the proposed ESMT algorithm ESMT-N if no energy constraints are involved (here, $\mathrm{N}$ denotes no energy consumption constraints). That is, ESMT$\mathrm{N}$ determines that $\mathrm{ONU}$ enters a deep sleep state only when $\Delta_{\text {idle }}>T_{\text {ov }}$. In addition, we take the previous algorithm in [18, 22] as comparison algorithm which focuses on energy saving oriented to multicast traffic in an EPON without upstream traffic. In simulation, we investigate the performance of our proposed algorithm and the above two algorithms on an EPON platform implemented by the OPNET simulator. On the EPON platform, the network topology is composed of an OLT, a splitter, and four ONUs about $25 \mathrm{~km}$ away from the OLT. In each ONU, there are two queues to buffer the upstream real-time and non-real-time packets, respectively. Similarly, in the OLT, there are two queues to buffer the downstream real-time and non-real-time packets for each ONU. We assume that these queues have infinite length and the packets queued in them are accessed by First In First Out (FIFO). The packet generation for both upstream and downstream cases follows a Poisson distribution. The average packet size is 500 bytes. For simplicity, we assume that each multicast group contains two ONUs at most, and the number of ONUs is generated randomly. $P_{\text {wake-up }}$ is computed as $0.48125 \mathrm{~mW}$ [4]. For other parameters, refer to [3].

5.1. Comparison of ESMT and ESMT-N [18, 22], Given an Upstream Packet Arrival Rate. Given an upstream packet arrival rate (10 packets $/ \mathrm{ms}$ ), we compare the four algorithms in terms of the average number of wake-up times (ANWT), average energy consumption (AEC) of each ONU, and the average packet delay of each downstream packet.

In Table 1, we show the four algorithms with an increasing downstream packet arrival rate. We can see that the ANWT of [18] is the smallest due to listen state, followed by ESMT and ESMT-N, and that of [22] is the largest. The result

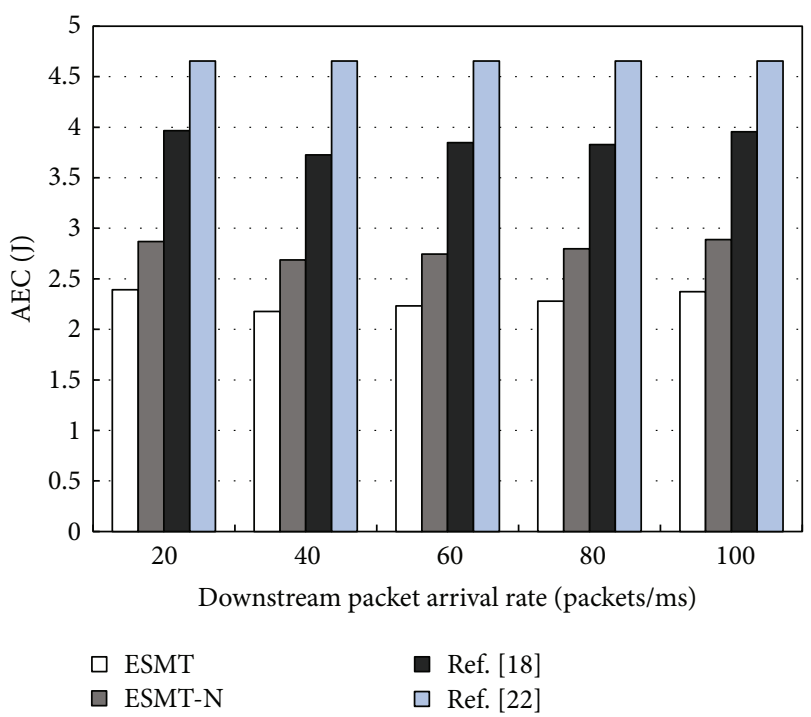

FIGURE 9: Comparison of AEC, given an upstream packet arrival rate of 10 packets $/ \mathrm{ms}$.

demonstrates the effectiveness of our proposed algorithm in terms of the reduction of wake-up times for each ONU. ANWT in [22] is constant because of the fixed sleep duration. Furthermore, the ANWT of ESMT is smaller than ESMT-N due to the additional constraints allowing ONUs to enter deep sleep state.

To highlight the advantage of ESMT over other algorithms, we show the AEC of the four algorithms in Figure 9. It is obvious that ESMT consumes the least energy. This is because the ANWT contributes a large part to the ONU energy consumption for the four algorithms. In addition to the ANWT, the AEC is also more dependent on the energy consumption of the ONUs' transmitters and receivers. Despite the presence of larger ANWT, ESMT do not include listen state which is more energy saving than deep sleep state, so ESMT is more energy efficient.

In Figure 10, we show the average packet delay of [18] and real-time delay (RD) and non-real-time delay (NRD) of ESMT, ESMT-N, and [22], as [18] does not consider the real-time performance of packets. Due to independent sleep mode in our proposed algorithm and [18], the packet delay outperforms that in [22].

From Table 1 and Figures 9 and 10, we can see that the proposed ESMT and ESMT-N algorithms outperform that in [22] in terms of wake-up times, energy consumption, and traffic delay. However, the cost is increased network overhead due to more GATE control messages. Nevertheless, the proposed algorithm is still more energy efficient. In other words, the effect produced by the increased network overhead is so little that it can be ignored in this paper. On the other hand, the proposed algorithm consumes less energy than that in [18] at the cost of packet delay, while they still meet QoS of the real-time packets.

5.2. Comparison of ESMT and ESMT-N, Given Two Different Upstream Packet Arrival Rates. In this subsection, we compare ESMT, ESMT-N, and [18] given two different upstream 
TABLE 1: Comparison of ANWT, given an upstream packet arrival rate of 10 packets/ms.

\begin{tabular}{lccccc}
\hline $\begin{array}{l}\text { Downstream packet } \\
\text { arrival rate (packets/ms) }\end{array}$ & 20 & 40 & 60 & 80 & 100 \\
\hline & ESMT & 297 & 440 & 443 & 297 \\
ANWT (times) & ESMT-N & 371 & 445 & 445 & 315 \\
& Reference [18] & 232 & 347 & 345 & 519 \\
& Reference [22] & 3432 & 3432 & 3432 & 349 \\
\hline
\end{tabular}

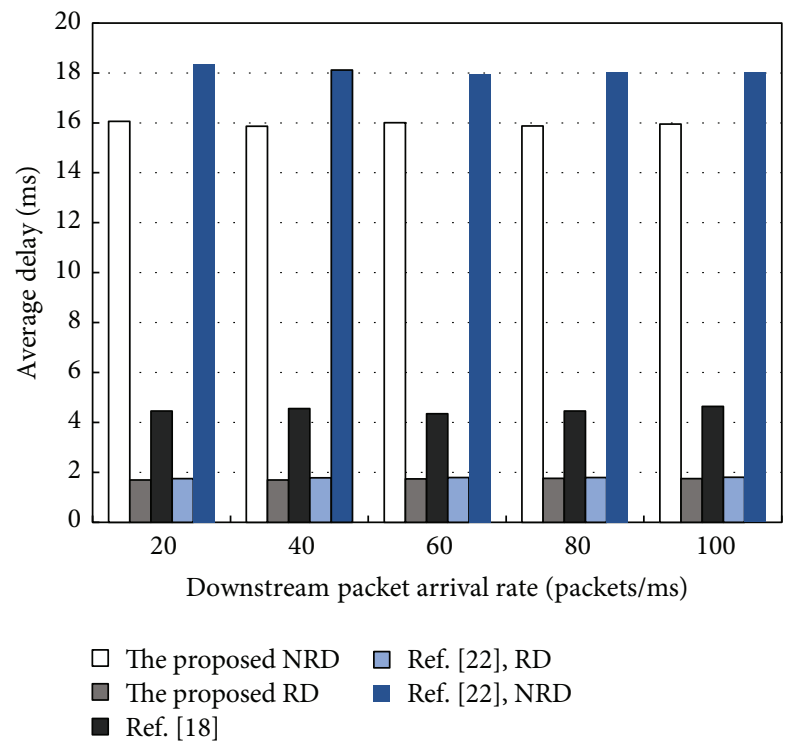

FIGURE 10: The average packet delay of the four algorithms given an upstream packet arrival rate of 10 packets $/ \mathrm{ms}$.

packet arrival rates (i.e., 10 packets/ms and 100 packets $/ \mathrm{ms}$ ) to study the influence of the upstream traffic load on the three algorithms. Since upstream traffic is not involved in [22], we do not consider it in this subsection. In addition to the ANWT and AEC, we also compare the AEC of the transmitter (AEC-T) and of the receiver (AEC-R) of the ONUs among ESMT, ESMT-N, and [18]. For simplicity, we define scenario 1 as having an upstream packet arrival rate of 10 packets $/ \mathrm{ms}$ and scenario 2 as having an upstream packet arrival rate of 100 packets/ms.

Figure 11 shows the ANWT of the three algorithms under two different upstream packet arrival rates. First, it is obvious that the ANWT of ESMT is smaller than ESMT-N, and the reason is the same as in Table 1 . In addition, when the downstream packet arrival rate is $20-80$ packets/ms, the ANWTs of ESMT and ESMT-N for scenario 2 are smaller than those for scenario 1. Thus, the increase of upstream traffic load does not increase the ONU wake-up times; however, the ANWT decreases conversely. The reason is that the increased upstream traffic load increases the probability of an upstream transmission stretching across two downstream transmission slots, where the ONU does not need to enter a deep sleep state. On the other hand, when the downstream packet arrival rate is 100 packets/ms, the ANWTs of ESMT and ESMT-N in scenario 2 are larger than those in scenario 1 . Here, the

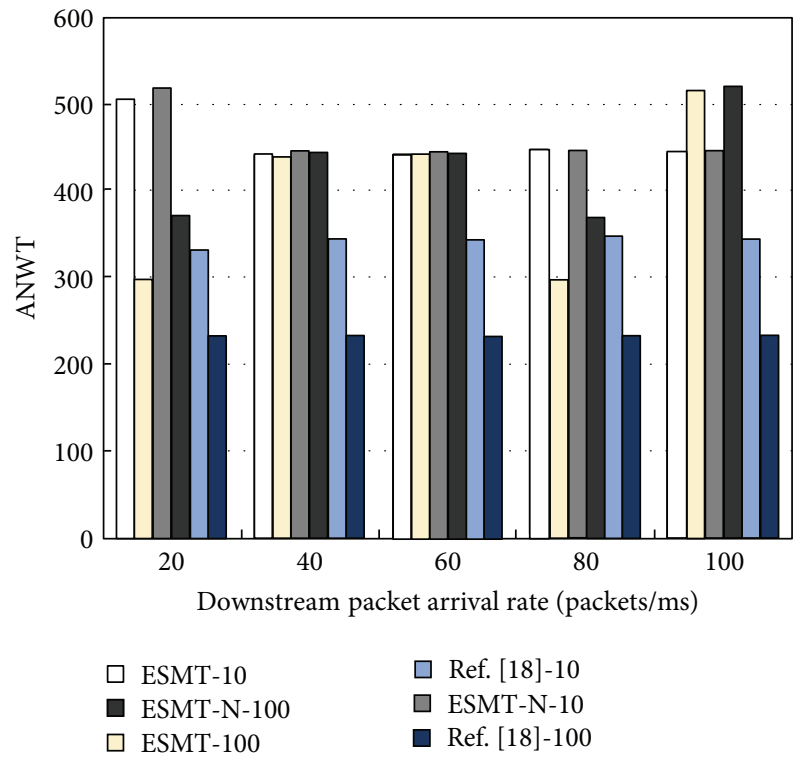

FIgURE 11: Comparison of ANWT under the two scenarios.

downstream traffic load is so large that it greatly reduces the probability of an upstream transmission stretching across two downstream transmission slots; therefore, the ONU wake-up times increase accordingly. In addition, compared with [18], the ANWTs of our proposed algorithm are much larger and the result is the same as in Table 1.

Figures 12 and 13, respectively, show the comparison of the AEC-T and AEC-R of each ONU under the two different upstream packet arrival rates. We can see that the changing trend of the AEC-T and AEC-R under the two scenarios is just the opposite of that of the ANWT in Figure 11. This is because the smaller the ONU wake-up times are, the longer the ONUs remain out of the deep sleep state where the transmitter (in Figure 12) and receiver (in Figure 13) are turned off. Therefore, the transmitter/receiver consumes more energy. The results also demonstrate that more energy is consumed in [18] in comparison to our proposed algorithm. It is worth noting that the energy consumption of transmitter and receiver increases with the traffic load in [18].

Figure 14 compares the AEC of the ONUs for ESMT, ESMT-N, and [18] under the two scenarios. The AEC of the ONUs is composed of AEC-T, AEC-R, and the energy consumed by the common modules and the wake-up processes. As is shown in Figure 14, under the same scenario, ESMT is more energy efficient than ESMT-N. Although the upstream traffic load of scenario 2 is larger than that of scenario 1 , the 


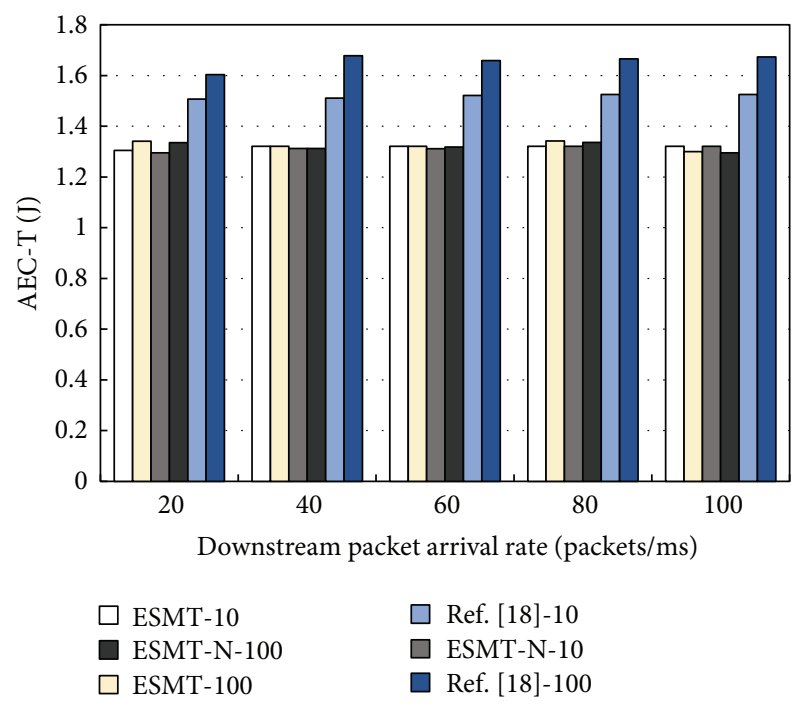

Figure 12: Comparison of AEC-T under the two scenarios.

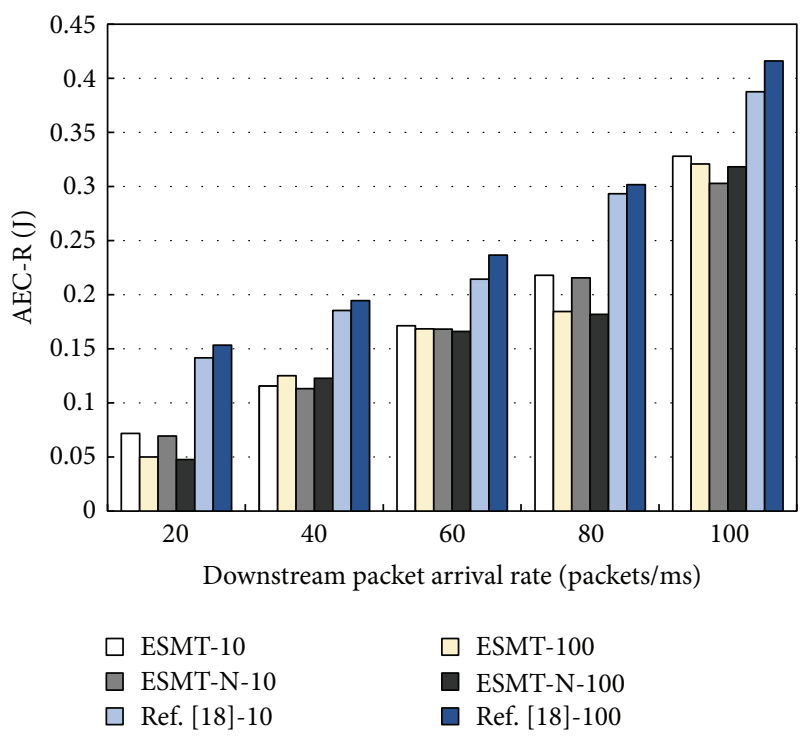

FIGURE 13: Comparison of AEC-R under the two scenarios.

AEC of both ESMT and ESMT-N does not always increase. Thus, it can be seen that increasing the upstream traffic load has a certain influence on the energy consumption of ESMT and ESMT-N but does not always increase them. The AEC of [18] is still larger than our proposed algorithm, which is in accord with the results in Section 5.1, and the increasing trend is in keeping with the energy consumption of the transmitter and receiver.

\section{Conclusion}

This paper proposed an ESMT algorithm to optimize the energy consumption of EPONs oriented to multicast traffic. In ESMT, the independent sleep mode and the deep sleep mode were combined with the characteristics of multicast traffic. Both upstream and downstream traffic loads were

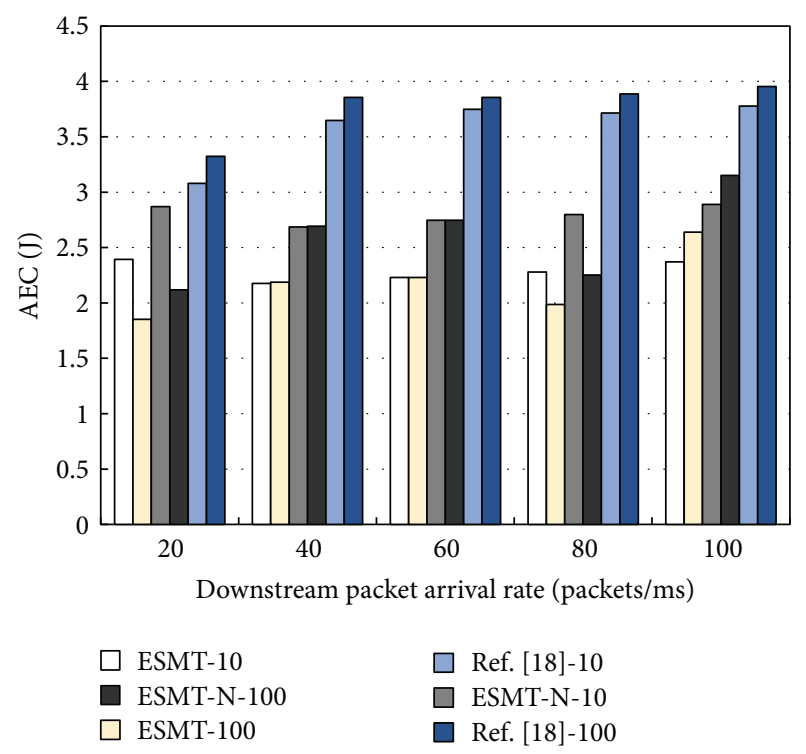

FIgURE 14: Comparison of AEC under the two scenarios.

considered when designing the energy-saving algorithm. In addition, the ONU wake-up times were also considered. The simulation was run from three different perspectives, and it was demonstrated that our proposed ESMT is effective in terms of both energy consumption and packet delay.

\section{Notations}

$P_{\mathrm{tx}}: \quad$ The power consumed by the transmitter of each ONU

$P_{\mathrm{rx}}: \quad$ The power consumed by the receiver of each $\mathrm{ONU}$

$P_{\text {sleep }}: \quad$ The power consumed by the common modules of each ONU

$P_{\text {wake-up }}$ : The power consumed by the wake-up process of each ONU

$t_{\mathrm{tx}}^{i}: \quad$ The transmission period of $\mathrm{ONU}_{i}$ 's

upstream traffic

$t_{\mathrm{rx}}^{i}: \quad$ The receiving period of $\mathrm{ONU}_{i}$ 's downstream traffic

$t_{\text {sleep }}^{i}$ : The deep sleep period of $\mathrm{ONU}_{i}$ 's downstream traffic

$t_{\text {sim }}^{i}$ : The number of wake-up times of $\mathrm{ONU}_{i}$

$n_{\text {wake-up }}^{i}$ : The running time of $\mathrm{ONU}_{i}$.

\section{Conflict of Interests}

The authors declare that there is no conflict of interests regarding the publication of this paper.

\section{Acknowledgments}

This work was supported by the National Natural Science Foundation of China (61501308), Liaoning BaiQianWan Talents Program (no. 2014921042), Key Laboratory Open 
Foundation of Shenyang Ligong University, and the Special Foundation of Doctoral Program of Shenyang Ligong University.

\section{References}

[1] J.-H. Jung, W.-H. Yang, and Y.-C. Kim, "A bandwidth allocation algorithm for improving QoS in EPON with sleep mode," in Proceedings of the 26th International Conference on Information Networking (ICOIN '12), pp. 324-328, IEEE, Bali, Indonesia, February 2012.

[2] Y. Yan, S.-W. Wong, L. Valcarenghi et al., "Energy management mechanism for ethernet passive optical networks (EPONs)," in Proceedings of the IEEE International Conference on Communications (ICC '10), pp. 1-5, Cape Town, South Africa, May 2010.

[3] L. Valcarenghi, D. P. Van, and P. Castoldi, "How to save energy in passive optical networks," in Proceedings of the 13th International Conference on Transparent Optical Networks (ICTON '11), pp. 1-5, IEEE, Stockholm, Sweden, June 2011.

[4] Y. Yan and L. Dittmann, "Energy efficiency in Ethernet Passive Optical Networks (EPONs): protocol design and performance evaluation," Journal of Communications, vol. 6, no. 3, pp. 249261, 2011.

[5] L. Zhang, Y. Liu, L. Guo, and X. Gong, "Energy-saving scheme based on downstream packet scheduling in Ethernet passive optical networks," Optical Fiber Technology, vol. 19, no. 2, pp. 169-178, 2013.

[6] J. Zhang and N. Ansari, "Toward energy-efficient 1G-EPON and 10G-EPON with sleep-aware MAC control and scheduling," IEEE Communications Magazine, vol. 49, no. 2, pp. S33-S38, 2011.

[7] G. Schütz and N. Correia, "Design of QoS-aware energyefficient fiber wireless access networks," Journal of Optical Communications and Networking, vol. 4, no. 8, pp. 586-594, 2012.

[8] A. R. Dhaini, P.-H. Ho, and G. Shen, "Toward green next-generation passive optical networks," IEEE Communications Magazine, vol. 49, no. 11, pp. 94-101, 2011.

[9] X. Gong, W. Hou, L. Zhang, Y. Liu, and L. Guo, "A novel energysaving algorithm based on sleeping low-loaded ONU for hybrid wireless-optical broadband access network," in Proceedings of the 2nd International Conference on Networking and Information Technology (ICNIT '11), pp. 335-342, Wuhan, China, May 2011.

[10] M. P. I. Dias and E. Wong, "Energy-efficient dynamic bandwidth allocation algorithm for sleep/doze mode VCSEL-ONU," in Proceedings of the Asia Communications and Photonics Conference (ACP '12), pp. 1-3, Guangzhou, China, November 2012.

[11] M. P. I. Dias and E. Wong, "Sleep/doze controlled dynamic bandwidth allocation algorithms for energy-efficient passive optical networks," Optics Express, vol. 21, no. 8, pp. 9931-9946, 2013.

[12] S.-W. Wong, L. Valcarenghi, S.-H. Yen, D. R. Campelo, S. Yamashita, and L. Kazovsky, "Sleep mode for energy saving PONs: advantages and drawbacks," in Proceedings of the IEEE GLOBECOM Workshops, pp. 1-4, Honolulu, Hawaii, USA, December 2009.

[13] S.-W. Wong, S.-H. Yen, P. Afshar, S. Yamashita, and L. G. Kazovsky, "Demonstration of energy conserving TDM-PON with sleep mode ONU using fast clock recovery circuit," in Proceedings of the Optical Fiber Communication Conference (OFC '10), Paper OThW7, pp. 1-3, San Diego, Calif, USA, March 2010.
[14] P. Chowdhury, M. Tornatore, S. Sarkar, and B. Mukherjee, "Building a green wireless-optical broadband access network (WOBAN)," Journal of Lightwave Technology, vol. 28, no. 16, Article ID 5424000, pp. 2219-2229, 2010.

[15] S. Yin, Y. Luo, N. Ansari, and T. Wang, "Stability of predictorbased dynamic bandwidth allocation over EPONs," IEEE Communications Letters, vol. 11, no. 6, pp. 549-551, 2007.

[16] ITU, “GPON power conservation,” ITU-T G.45, 2009.

[17] J. Zhang, M. T. Hosseinabadi, and N. Ansari, "Standardscompliant EPON sleep control for energy efficiency: design and analysis," Journal of Optical Communications and Networking, vol. 5, no. 7, Article ID 6560469, pp. 677-685, 2013.

[18] M. Hosseinabadi and N. Ansari, "Multi-power-level energy saving management for passive optical networks," Journal of Optical Communications and Networking, vol. 6, no. 11, Article ID 6954279, pp. 965-973, 2014.

[19] N. Kim, H.-S. Lim, and M. Kang, "Fair bandwidth allocation using effective multicast traffic share in TDM-PONs," Journal of Lightwave Technology, vol. 26, no. 7, pp. 756-767, 2008.

[20] N. Kim and M. Kang, "Traffic share-based multicast scheduling for broadcast video delivery in shared-WDM-PONs," Journal of Lightwave Technology, vol. 25, no. 9, pp. 2814-2827, 2007.

[21] M. Alfani, J. M. Finochietto, and F. Neri, "Efficient multicast bandwidth allocation in TDM-WDM PONs," in Proceedings of the Conference on Optical Fiber Communication-Incudes Post Deadline Papers (OFC '09), pp. 1-3, San Diego, Calif, USA, March 2009.

[22] L. Valcarenghi and P. Castoldi, "Impact of unicast and multicast traffic on ONU energy savings," in Proceedings of the 14th International Conference on Transparent Optical Networks (ICTON '12), pp. 1-5, Coventry, UK, July 2012.

[23] L. Valcarenghi, I. Cerutti, and P. Castoldi, "Energy efficient multicasting in TDMA PONs," Journal of High Speed Networks, vol. 18, no. 4, pp. 213-222, 2011.

[24] S. H. S. Newaz, A. Cuevas, G. M. Lee, N. Crespi, and J. K. Choi, "Evaluating energy efficiency of ONUs having multiple power levels in TDM-PONs," IEEE Communications Letters, vol. 17, no. 6, pp. 1248-1251, 2013. 


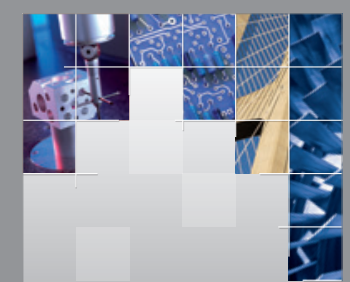

\section{Enfincering}
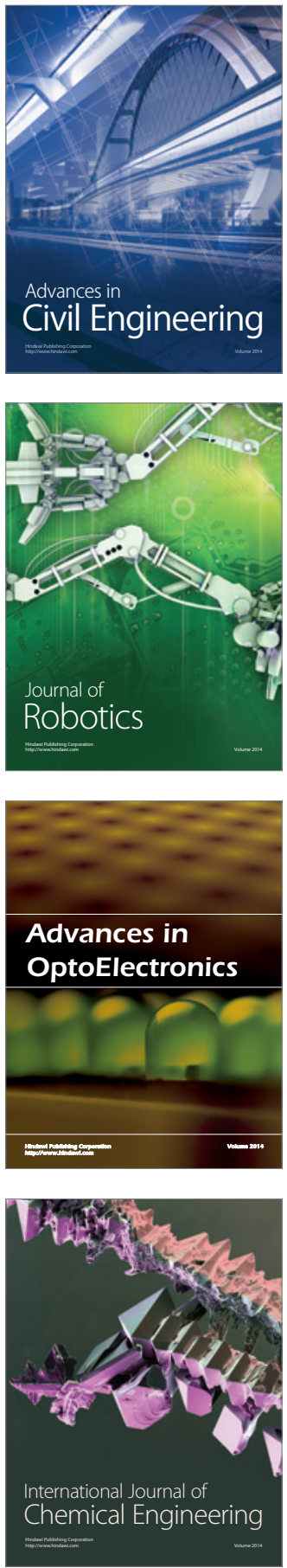

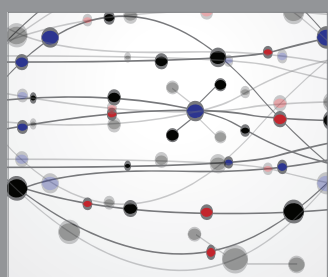

The Scientific World Journal

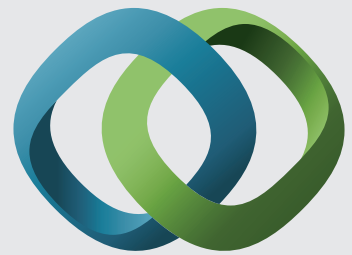

\section{Hindawi}

Submit your manuscripts at

http://www.hindawi.com
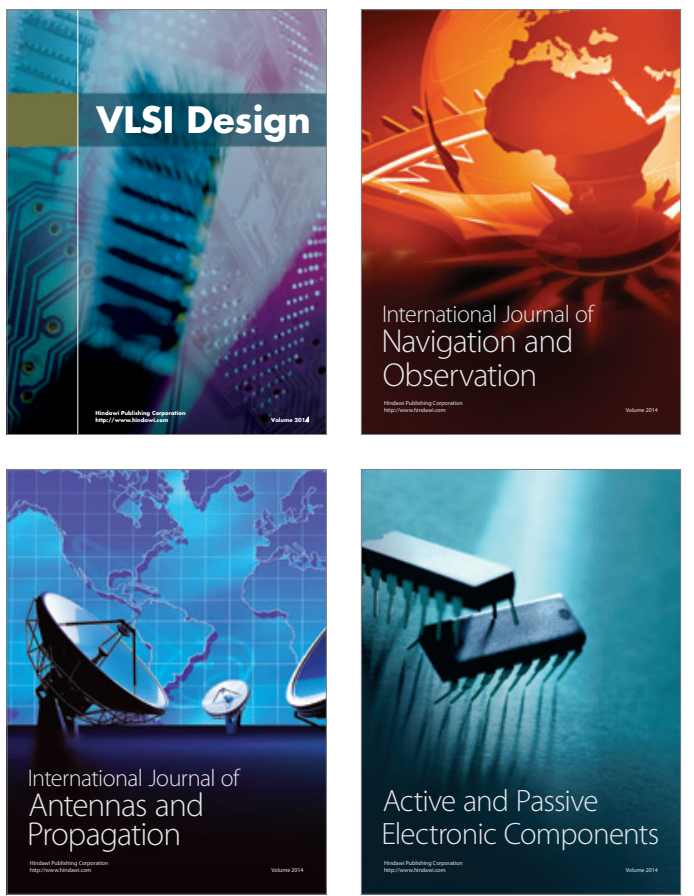
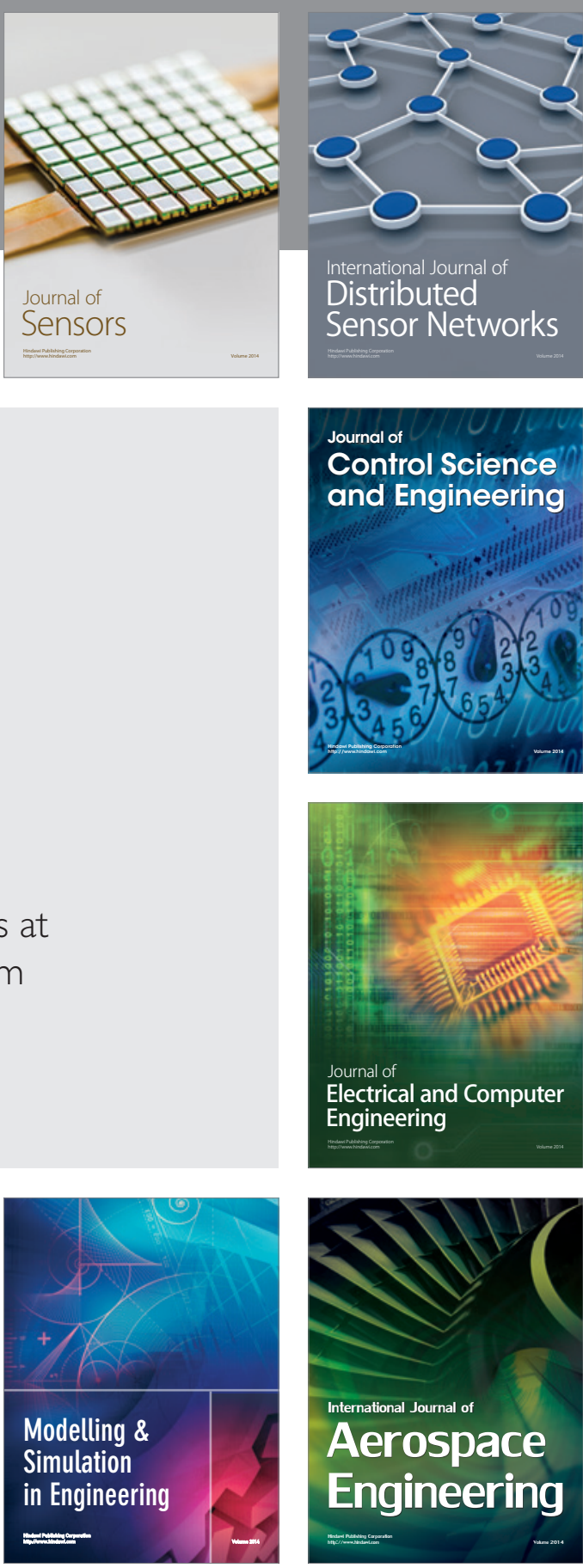

International Journal of

Distributed

Sensor Networks

Journal of

Control Science

and Engineering
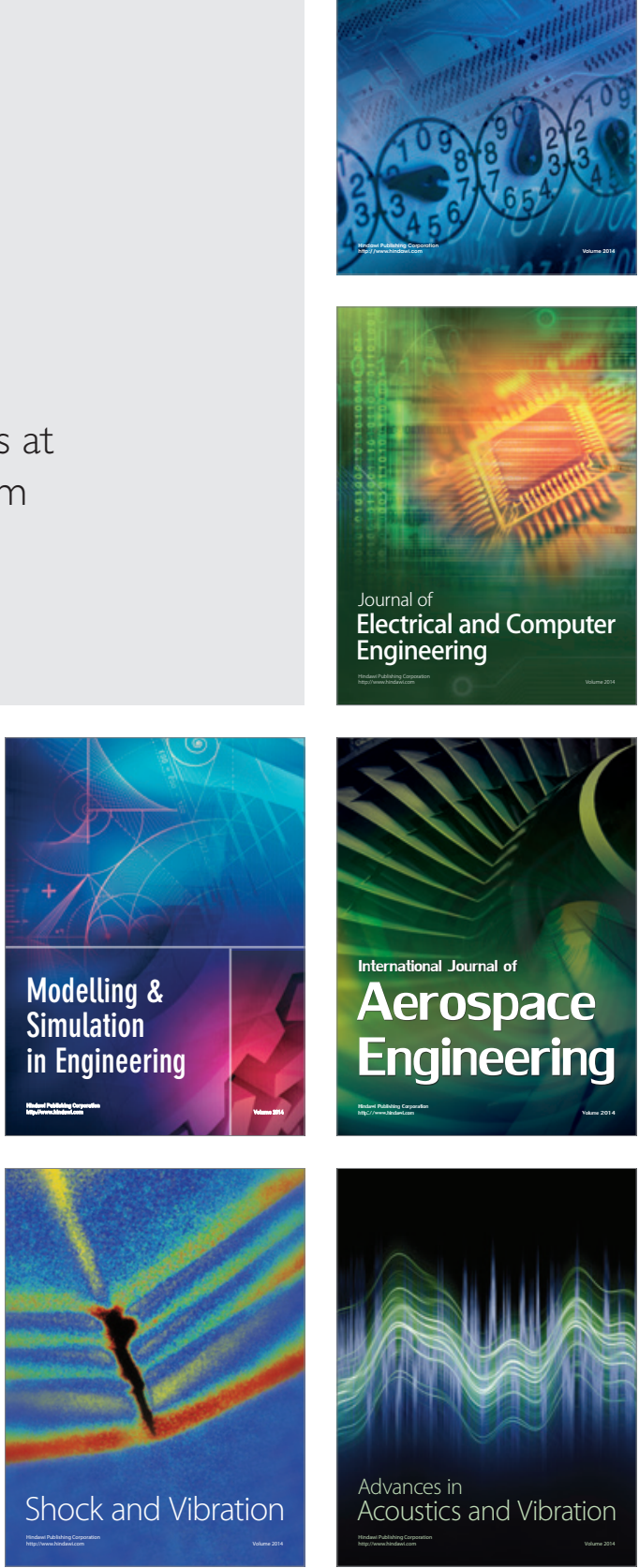